\title{
Development and validation of a delirium risk assessment tool in older patients admitted to the Emergency Department Observation Unit
}

\author{
A. Zucchelli ${ }^{1}$ (D) R. Apuzzo ${ }^{2} \cdot$ C. Paolillo ${ }^{3} \cdot$ V. Prestipino ${ }^{4} \cdot$ S. De Bianchi ${ }^{5} \cdot$ G. Romanelli ${ }^{6} \cdot$ A. Padovani $^{6}$. \\ A. Marengoni ${ }^{6} \cdot$ G. Bellelli ${ }^{7,8}$
}

Received: 27 November 2020 / Accepted: 11 January 2021 / Published online: 9 February 2021

(c) The Author(s) 2021

\begin{abstract}
Background Delirium is frequent though undetected in older patients admitted to the Emergency Department (ED).

Aims To develop and validate a delirium risk assessment tool for older persons admitted to the ED Observation Unit (OU). Methods We used data from two samples of $65+$ year-old patients, one admitted to the ED of Brescia Hospital $(n=257)$ and one to the ED of Desio Hospital ( $n=107)$, Italy. Data from Brescia were used as training sample, those collected in Desio as testing one. Delirium was assessed using the 4AT and patients' characteristic were retrieved from medical charts. Variables found to be associated with delirium in the training sample were tested for the creation of a delirium risk assessment tool. The resulting tool's performances were assessed in the testing subsample.

Results Of all possible scores tested, the combination with the highest discriminative ability in the training sample included: age $\geq 75$ years, dementia diagnosis, chronic use of neuroleptics, and hearing impairment. The delirium score exhibited an AUC of 0.874 and 0.893 in the training and testing samples, respectively. For a 1-point increase in the score, the odds of delirium increased more than twice in both samples.

Discussion We propose a delirium risk assessing tool that includes variables that can be easily collected at ED admission and that can be calculated rapidly.
\end{abstract}

Conclusion A risk assessment tool could help improving delirium detection in older persons referring to ED.

Keywords Delirium $\cdot$ Prediction score $\cdot$ Older persons $\cdot$ Emergency department

A. Marengoni and G. Bellelli have Co-last authorship.

A. Zucchelli

a.zucchelli001@unibs.it

1 Department of Information Engineering, University of Brescia, v. Branze, 38, 25123 Brescia, Italy

2 SC Medicina Generale, Ospedale Sant'Andrea di Vercelli, ASL VC, Vercelli, Italy

3 Emergency Department, ASST Spedali Civili di Brescia, Brescia, Italy

4 Department of Clinical and Experimental Medicine, University of Messina, Messina, Italy

5 Emergency Department, Desio Hospital, ASST Monza e Brianza, Desio, Italy

6 Department of Clinical and Experimental Science, University of Brescia, Brescia, Italy

7 University of Milano-Bicocca, Milan, Italy

8 Geriatric Unit, San Gerardo Hospital, Monza, Italy

\section{Background}

Delirium is a neuropsychiatric disorder, characterized by acute onset and fluctuating course, change in awareness, arousal, and other cognitive dysfunctions [1]. Delirium is a frequent feature of many acute medical conditions or drug's intoxication and increases several negative outcomes, including mortality, morbidity, functional impairment [2,3], and distress of patients and caregivers $[4,5]$.

The availability of simple scores to predict delirium occurrence in first-intervention settings, such as the Emergency Department (ED), may be particularly useful. Indeed, prompt recognition of delirium is crucial: its misrecognition can lead to inadequate diagnostic evaluations, inappropriate dispositions and diagnosis delays $[6,7]$ and delirium that is missed in the ED is frequently missed in the acute hospital wards too $[8,9]$. 
However, to date, there are only two studies that have proposed non-externally validated scores to predict delirium in $\operatorname{ED}[10,11]$.

The aim of this study was to develop and validate a delirium score for older persons admitted to the ED Observation Unit (OU).

\section{Methods}

We retrospectively collected data from two samples of patients, one admitted to the ED of the Civili Hospital in Brescia $(n=257)$ and the other to the ED of the Desio Hospital (Monza) $(n=107)$, Italy. Data were collected between November 2018 and February 2019 in Brescia and between July and September 2019 in Desio. To decrease the risk of overfitting and to test the generalizability of the proposed score, the first sample (Brescia) was employed to build the delirium risk assessment tool (training sample), whereas the second one (Desio) was used to externally validate the chosen score and to test its accuracy (testing sample).

The only inclusion criterium was age equal or above 65 years; exclusion criteria were coma, end-stage dementia and inability to speak Italian.

Among patients evaluated, 9 were excluded for missing information on most of the variables.

The study was approved by the Ethical Committee of the Brescia County and the one of Monza.

\section{Diagnosis of delirium}

Presence of delirium was assessed by the attending physician and a resident in geriatrics using the 4AT test [12]. The 4AT has shown excellent sensitivity and specificity to diagnose delirium at a threshold score of $\geq 4[12,13]$. The 4 AT was repeated every eight hours: delirium was considered present if a score $\geq 4$ was exhibited in at least one evaluation.

\section{Data collection}

The following data were retrieved from medical charts: reason for ED admission, vital parameters, presence of visual and/or hearing loss, and chronic conditions. A patient was considered affected by dementia if an established diagnosis of dementia was reported in his/her medical history. The pain was measured with a numerical rating scale. Laboratory tests were performed in each patient.

Presence of "suspected infection" was based on the reason of ED admission and/or C-reactive protein levels higher than $5 \mathrm{mg} / \mathrm{L}$.

Patients' chronic therapy was retrieved from medical records and in categories. Antiepileptic drugs, antipsychotics, antidepressants (SNRI, SSRI, atypical, tricyclics), anti-dementia drugs, hypnotics, opioids, and drugs for Parkinson disease were grouped in a single category named "psychotropics".

The total length of stay in OU was recorded as well as the number of patients who spent $2+$ hours in OU between 06:00 $\mathrm{pm}$ and 06:00 am.

\section{Statistical analyses}

The differences between the training (Brescia's ED) and the testing (Desio's ED) sample were investigated employing chi-squared tests or exact e Fisher's tests and $t$-tests or Mann-Whitney tests, as appropriate. The association between patient's characteristics and delirium was explored by means of unadjusted logistic regression models in the training sample. We used a $p \leq 0.200$ cut-off to select the variables associated with delirium to be tested for the creation of the delirium risk assessment tool. Continuous variables associated with delirium were dichotomized using a clinically suitable cut-off.

The following principles were used to create the score: (1) the final tool should have included between 2 and 4 variables and (2) a score between 1 and 3 could have been assigned to each included variable. All possible combinations of variables, number of variables included, and points assigned were tested. The discriminative ability to predict delirium in the training sample was evaluated using the area under the curve (AUC) obtained from non-parametric Receiver-OperatingCharacteristic (ROC) analyses. The combination showing the highest AUC in the training sample was selected and the performances of the resulting score were assessed in the testing sample. All analyses were conducted with R 4.0.0 (R Core Team-R Foundation for Statistical Computing, Vienna, Austria).

\section{Results}

The characteristics of the training and testing samples are described in Table S1. The proportion of patients who were diagnosed with delirium was $16.1 \%$ in the training sample and $34.6 \%$ in the testing sample. In the latter, patients were older, more likely to have dementia, more likely to be admitted for a suspected infectious disease and spent more time in the OU in comparison with those in the training sample.

Seven variables were found to be associated with delirium in the training sample and were considered for the creation of the delirium score (Table 1). When evaluated alone, each drug class included in the "psychotropics" group was found positively associated with delirium, although 4 of them (i.e. antiepileptics - proportion $=2.5 \%$, atypical antidepressants $-0.8 \%$, opioids $-6.1 \%$, and tricyclics $-1.1 \%$ ) did 
Table 1 Associations (odds ratios-OR—and 95\% confidence intervals-95\% CI) of patients' characteristics with delirium development and their area under the curve (AUC), in the training sample

\begin{tabular}{|c|c|c|c|}
\hline & OR $(95 \% \mathrm{CI})$ & $p$ & AUC \\
\hline Age & $1.12(1.06-1.17)$ & $<0.001$ & 0.733 \\
\hline Age $\geq 75$ years old & $9.22(3.20-39.06)$ & $<0.001$ & 0.676 \\
\hline Male sex & $0.89(0.44-1.76)$ & 0.744 & 0.514 \\
\hline Living in nursing home & $2.03(0.43-7.38)$ & 0.313 & 0.518 \\
\hline Dementia & $18.33(8.08-43.64)$ & $<0.001$ & 0.744 \\
\hline Hearing impairments & $8.07(3.80-17.44)$ & $<0.001$ & 0.699 \\
\hline Visual impairments & $1.03(0.45-2.21)$ & 0.932 & 0.503 \\
\hline Anticoagulant drugs & $1.4(0.61-3.02)$ & 0.406 & 0.529 \\
\hline PPIs & $1.47(0.74-2.98)$ & 0.272 & 0.548 \\
\hline Insulin & $1.42(0.45-3.81)$ & 0.511 & 0.517 \\
\hline NSAIDs & $1.32(0.19-5.5)$ & 0.735 & 0.506 \\
\hline Opioids & $1.61(0.35-5.54)$ & 0.488 & 0.513 \\
\hline Psychotropics & $5.55(2.68-12.23)$ & $<0.001$ & 0.728 \\
\hline Suspected Infection & $2.23(1.12-4.45)$ & 0.022 & 0.597 \\
\hline Cardiovascular event & $1.56(0.73-3.21)$ & 0.234 & 0.545 \\
\hline Stroke & $5.31(0.21-136.13)$ & 0.241 & 0.51 \\
\hline Acute urinary retention & $0.38(0.02-2.02)$ & 0.364 & 0.519 \\
\hline Heart rate & $1.00(0.98-1.02)$ & 0.899 & 0.503 \\
\hline Systolic blood pressure & $0.98(0.96-0.99)$ & 0.010 & 0.628 \\
\hline Systolic blood pressure $<120 \mathrm{mmHg}$ & $2.49(0.98-6.00)$ & 0.049 & 0.554 \\
\hline Peripheral arterial blood oxygen saturation & $1.00(0.9-1.16)$ & 0.976 & 0.531 \\
\hline Pain & $1.00(0.89-1.13)$ & 0.938 & 0.506 \\
\hline Haemoglobin $<10 \mathrm{~g} / \mathrm{dL}$ & $1.25(0.34-3.61)$ & 0.704 & 0.509 \\
\hline Creatinine & $1.1(0.81-1.43)$ & 0.467 & 0.624 \\
\hline Sodium & $0.94(0.87-1.01)$ & 0.092 & 0.630 \\
\hline Sodium $<135 \mathrm{mEq} / \mathrm{L}$ & $2.54(0.92-6.47)$ & 0.057 & 0.549 \\
\hline Total time spent & $1.01(0.97-1.04)$ & 0.660 & 0.529 \\
\hline $2+$ hours spent between 06:00 pm and 06:00 am & $1.84(0.68-6.44)$ & 0.274 & 0.535 \\
\hline
\end{tabular}

Variables with a $p<=0.200$ were tested for the creation of the delirium risk assessment score PPIs proton pumps inhibitors, NSAIDs non-steroidal anti-inflammatory drugs not reach statistical significance (data not shown). In total, 3969 possible scores were tested in the training sample. The combination that lead to the highest discriminative ability in the training sample included age $\geq 75$ years old ( 2 points), dementia diagnosis ( 3 points), chronic use of psychotropic drugs ( 1 point), and hearing impairment (2 point) (Fig. 1). In both samples, the score ranged between 0 and 8 points and the median value was 2 points.

Figure 2 and Table S2 show the performance of the delirium score. The score exhibited an AUC of 0.874 and 0.893 in the training and testing samples, respectively. In both samples, a cut-off of 3 or more points identified delirium with a sensitivity higher than 0.80 , while a cut-off of 5 or more points leads to a specificity of 0.90 or higher. For a 1-point increase in the score, the odds of developing delirium increased more than twice in both samples [training sample OR (95\%CI): 2.22 (1.80-2.84); testing sample OR (95\%CI): 2.35 (1.78-3.30)]. After excluding patients with a diagnosis of dementia, the odds ratios of developing delirium for a 1-point increase were 2.17 (95\% CI: $1.53-3.22)$ and 2.70 (95\% CI: 1.52-5.37) in the training and testing sample, respectively.

\section{Discussion}

We developed and validated a delirium risk assessment tool using retrospective data from two different samples of older patients admitted to the ED OU. Variables included in the score were age $\geq 75$ years old, dementia, hearing impairment, and chronic use of psychotropic drugs. In both samples, a cut-off of 3 or more points identified delirium with a sensitivity higher than 0.80 , while a cut-off of 5 or more points lead to a specificity of 0.90 or higher.

Age, dementia, and hearing impairment have been shown to be associated with delirium in previous studies $[14,15]$. Further, several drugs of those included in the "psychotropics" group have been directly associated with 
Fig. 1 Summary of the components of the delirium risk assessment tool

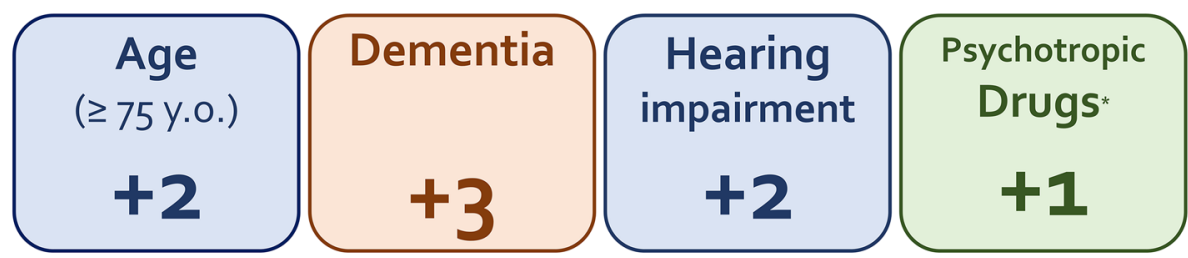

\title{
$\geq 3$ points
}

HIGH RISK OF DELIRIUM

\begin{abstract}
*At least one chronically prescribed drug among: antidepressants (SSRI, SNRI, tryciclic, atypical), antipsychotics (atypical and typical), benzodiazepines, trazodone, opioids, antiepileptics, anti-dementia, and drug for Parkinson's disease
\end{abstract}

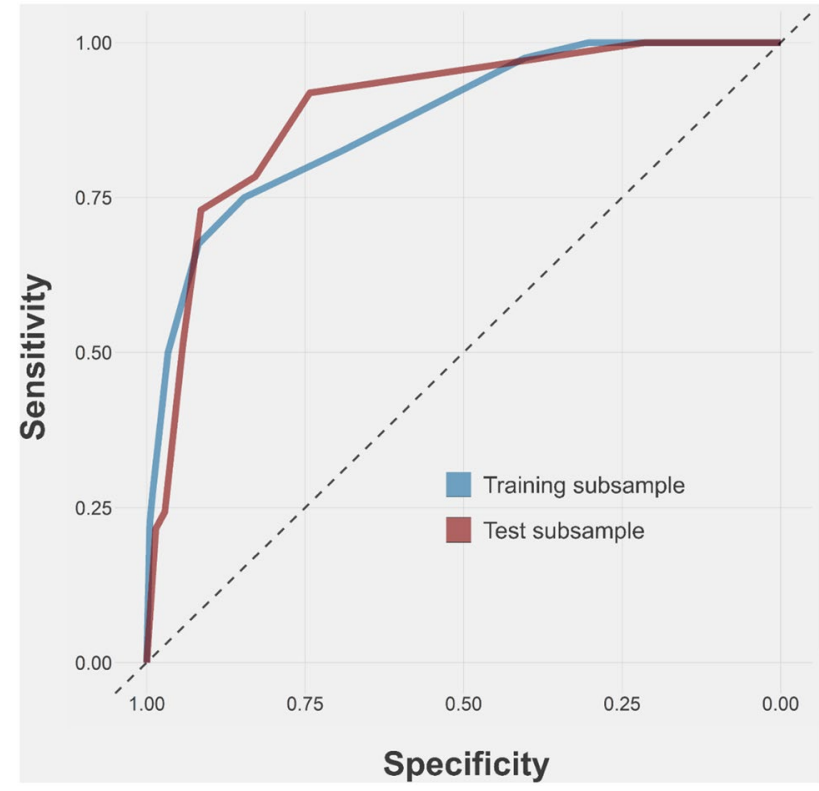

Fig. 2 Delirium predictive score's ROC curves (training and testing samples)

an increased risk of delirium in the literature $[16,17]$. However, it is also likely that the chronic prescription of psychotropic drugs reflects the presence of conditions that may increase the chances of delirium, such as previous episodes of delirium, cognitive impairment or dementia, explaining the increase in the discriminative capacity of the score after the inclusion of chronic psychotropic therapy among the variables.

Other scores have been proposed. The delirium risk score by Han et al. [18] was validated against the Confusion
Assessment Method (CAM) for the Intensive Care Unit [19] demonstrating good performance $(\mathrm{AUC}=0.82)$ and included three variables: hearing impairment, dementia, and KatzActivity of Daily Living (ADL). The risk prediction rule by Kennedy et al. [10] consisted of 5 variables (i.e. older age, history of stroke, dementia, suspected infection, tachypnea, and intracranial hemorrhage) and demonstrated fair prediction ability towards a CAM-defined diagnosis of delirium (AUC $=0.77$ ). The score by Pendlebury et al. [20] was created to identify both prevalent and incident delirium in acute wards and included older age, cognitive impairment, severe illness, infection, and visual impairment. The AUC of this score was 0.78 for any form of delirium, as defined with the CAM and DSM-IV criteria. Limits of these scores are that they were not externally validated [10], that delirium was assessed only at a single time-point in the ED [10] and that they require additional scales to be computed [11]. Furthermore, two studies used delirium diagnostic tools that are not applicable in drowsy patients $[10,20]$ which may have resulted in an underestimation of hypoactive delirium, which is the commonest subtype in ED [11].

The delirium score we propose has several advantages; first, it includes variables that can be easily collected at ED admission by the attending physician or nurse irrespectively of his/her expertise. Second, the score can be calculated rapidly, characteristic that is of most importance in critical care settings.

Given the frequent underdiagnosis of delirium in the EDs [11], a delirium risk assessment tool could help to improve delirium detection in older persons referring to acute care. The early identification of persons at increased risk of developing delirium in OU may help to put in place preventive strategies to decrease the chances of delirium, as well as to 
prompt a comprehensive geriatric assessment that may help to recognize possible triggers and causes of delirium.

\section{Limitations}

The results of our study should be read in light of some limitations: first, data about the timing of delirium development (i.e. as presentation of an acute disease, during the evaluation in the ED or during the OU staying) were not available; however, all variables included in our score were retrieved from the past medical history of the patient and are not subject to delirium onset's timing; furthermore, we selected the combination of variables showing the highest AUC in the training dataset, increasing the risk of overfitting: however, the association of all selected variables with delirium is known and the score was validated in another sample. Future studies are needed in order to investigate the causal relationship between the variables included in our tool and delirium; lastly, dementia alone showed a high discriminative ability in the prediction of delirium, however almost $40 \%$ of the patients developing delirium missed a previous dementia diagnosis and our score was strongly associated with delirium development in patients without known cognitive impairment.

\section{Conclusion}

A simple and rapid risk assessment tool could help improving delirium detection in older persons referring to ED. Supplementary Information The online version contains supplementary material available at https://doi.org/10.1007/s40520-021-01792-4.

Author contributions Conception and design of the work: AZ, AM, GB. Data collection: RA, VP. Data analysis: AZ. Interpretation of the results: all coauthors. Drafting the article: AZ, AM, GB. Critical revision of the manuscript: all coauthors. Final approval of the manuscript: all coauthors. All the authors fulfil the ICMJE criteria for authorship.

Funding Open Access funding provided by Università degli Studi di Brescia. The authors received no financial support for the research, authorship, and/or publication of this article.

Availability of data and materials Access to the original data is available to the research community upon approval by the data management committee. Application for accessing data can be sent to: alessandra. marengoni@unibs.it and giuseppe.bellelli@unimib.it.

\section{Compliance with ethical standards}

Conflict of interests All authors declare that no organizations have attempted to influence the study or the writing of the manuscript. They have had no financial relationships in the previous three years with any organizations that might have an interest in influencing the submitted work and no other relationships or activities that could appear to have influenced the submitted work.
Ethical approval The study was approved by the Ethical Committee of the Brescia County and the one of Monza. All procedures performed in this study involving human participants were in accordance with the1964 Helsinki declaration and its later amendments.

Human and animal rights This retrospective chart review study involving human participants was in accordance with the ethical standards of the institutional and national research committee and with the 1964 Helsinki Declaration and its later amendments or comparable ethical standards. The Ethical Committees of Brescia and Monza approved this study.

Informed consent Informed consent was obtained from all participants. In case of delirium or severe cognitive decline, the informed consent was signed by a proxy.

Transparency statement The lead author affirm that the manuscript is an honest, accurate, and transparent account of the study being reported; that no important aspects of the study have been omitted; and that any discrepancies from the study as originally planned have been explained.

Open Access This article is licensed under a Creative Commons Attribution 4.0 International License, which permits use, sharing, adaptation, distribution and reproduction in any medium or format, as long as you give appropriate credit to the original author(s) and the source, provide a link to the Creative Commons licence, and indicate if changes were made. The images or other third party material in this article are included in the article's Creative Commons licence, unless indicated otherwise in a credit line to the material. If material is not included in the article's Creative Commons licence and your intended use is not permitted by statutory regulation or exceeds the permitted use, you will need to obtain permission directly from the copyright holder. To view a copy of this licence, visit http://creativecommons.org/licenses/by/4.0/.

\section{References}

1. American Psychiatric Association, American Psychiatric Association. DSM-5 Task Force (2013) Diagnostic and statistical manual of mental disorders: DSM-5. 5th edn. American Psychiatric Association, Washington, D.C.

2. Morandi A, Mazzone A, Bernardini B et al (2019) Association between delirium, adverse clinical events and functional outcomes in older patients admitted to rehabilitation settings after a hip fracture: a multicenter retrospective cohort study. Geriatr Gerontol Int 19:404-408. https://doi.org/10.1111/ggi.13628

3. Morandi A, Davis D, Fick D et al (2014) Delirium superimposed on dementia strongly predicts worse outcomes in older rehabilitation inpatients. J Am Med Dir Assoc 15:349-354. https://doi. org/10.1016/j.jamda.2013.12.084

4. Morandi A, Lucchi E, Turco R et al (2015) Delirium superimposed on dementia: a quantitative and qualitative evaluation of informal caregivers and health care staff experience. J Psychosom Res 79:272-280. https://doi.org/10.1016/j.jpsychores.2015.06.012

5. Morandi A, Lucchi E, Turco R et al (2015) Delirium superimposed on dementia: a quantitative and qualitative evaluation of patient experience. J Psychosom Res 79:281-287. https://doi. org/10.1016/j.jpsychores.2015.07.010

6. Boucher V, Lamontagne ME, Nadeau A et al (2019) Unrecognized incident delirium in older emergency department patients. J Emerg Med 57:535-542. https://doi.org/10.1016/j.jemer med.2019.05.024 
7. Kakuma R, du Fort G, Arsenault L et al (2003) Delirium in older emergency department patients discharged home: effect on survival. J Am Geriatr Soc 51:443-450. https://doi.org/10.104 6/j.1532-5415.2003.51151.x

8. Han JH, Eden S, Shintani A et al (2011) Delirium in older emergency department patients is an independent predictor of hospital length of stay. Acad Emerg Med 18:451-457. https://doi.org/10.1 111/j.1553-2712.2011.01065.x

9. Han JH, Wilson A, Vasilevskis EE et al (2013) Diagnosing delirium in older emergency department patients: validity and reliability of the delirium triage screen and the brief confusion assessment method. Ann Emerg Med 62:457-465. https://doi. org/10.1016/j.annemergmed.2013.05.003

10. Kennedy M, Enander RA, Tadiri SP et al (2014) Delirium risk prediction, healthcare use and mortality of elderly adults in the emergency department. J Am Geriatr Soc 62:462-469. https://doi. org/10.1111/jgs. 12692

11. Han JH, Zimmerman EE, Cutler N et al (2009) Delirium in older emergency department patients: recognition, risk factors, and psychomotor subtypes. Acad Emerg Med 16:193-200. https://doi.org /10.1111/j.1553-2712.2008.00339.x

12. Bellelli G, Morandi A, Davis D et al (2014) Validation of the 4AT, a new instrument for rapid delirium screening: a study in 234 hospitalised older people. Age Ageing 43:496-502. https:// doi.org/10.1093/ageing/afu021

13. Shenkin SD, Fox C, Godfrey M et al (2019) Delirium detection in older acute medical inpatients: a multicentre prospective comparative diagnostic test accuracy study of the 4AT and the confusion assessment method. BMC Med 17:138. https://doi.org/10.1186/ s12916-019-1367-9

14. Ahmed S, Leurent B, Sampson EL (2014) Risk factors for incident delirium among older people in acute hospital medical units: a systematic review and meta-analysis. Age Ageing 43:326-333. https://doi.org/10.1093/ageing/afu022

15. Morandi A, Inzitari M, Udina C, et al for the Italian Study Group of Delirium (2020) Visual and hearing impairment are associated with delirium in hospitalized patients: results of a multisite prevalence study. J Am Med Dir Assoc. https://doi.org/10.1016/j. jamda.2020.09.032

16. Flacker JM, Cummings V, Mach JR Jr et al (1998) The association of serum anticholinergic activity with delirium in elderly medical patients. Am J Geriatr Psychiatry 6:31-41

17. Aloisi G, Marengoni A, Morandi A et al (2019) Drug prescription and delirium in older inpatients: results from the nationwide multicenter Italian delirium day 2015-2016. J Clin Psychiatry. https://doi.org/10.4088/JCP.18m12430

18. Han J, Shintani A, Eden S et al (2009) Delirium in the emergency department: an independent predictor of death within 6 months. Acad Emerg Med 16:193-200

19. Ely EW, Inouye SK, Bernard GR et al (2001) Delirium in mechanically ventilated patients: validity and reliability of the confusion assessment method for the intensive care unit (CAM-ICU). JAMA 286:2703-2710

20. Pendlebury ST, Lovett NG, Smith SC et al (2017) Delirium risk stratification in consecutive unselected admissions to acute medicine: validation of a susceptibility score based on factors identified externally in pooled data for use at entry to the acute care pathway. Age Ageing 46:226-231. https://doi.org/10.1093/ageing/afw198

Publisher's Note Springer Nature remains neutral with regard to jurisdictional claims in published maps and institutional affiliations. 